\title{
Probable person to person transmission of novel avian influenza A (H7N9) virus in Eastern China, 2013: epidemiological investigation
}

\author{
(c) $\stackrel{(1)(8)}{\text { ay }}$ OPEN ACCESS
}

Xian Qi viriologist ${ }^{1}$, Yan-Hua Qian epidemiologist ${ }^{2}$, Chang-Jun Bao epidemiologist ${ }^{1}$, Xi-Ling Guo microbiologist $^{3}$, Lun-Biao Cui molecular biologist ${ }^{3}$, Fen-Yang Tang public health officer ${ }^{1}$, Hong Ji public health officer ${ }^{1}$, Yong Huang trainee of CFETP ${ }^{4}$, Pei-Quan Cai respiratory physician ${ }^{5}$, Bing Lu deputy director ${ }^{2}$, Ke Xu public health officer ${ }^{1}$, Chao Shi public health officer ${ }^{2}$, Feng-Cai Zhu professor $^{6}$, Ming-Hao Zhou director ${ }^{6}$, Hua Wang epidemiologist and deputy director-general ${ }^{67}$

${ }^{1}$ Department of Acute Infectious Disease Control and Prevention, Jiangsu Province Center for Disease Control and Prevention, Nanjing, Jiangsu, China 210009; ${ }^{2}$ Wuxi Municipal Centre for Disease Control and Prevention, Wuxi, Jiangsu, China $214023 ;{ }^{3}$ Key Lab of Enteric Pathogenic Microbiology (Ministry of Health), Institute of pathogenic microbiology, Jiangsu Provincial Center for Disease Control and Prevention, Nanjing, Jiangsu, China 210009; ${ }^{4}$ Chinese Field Epidemiology Training Program, Beijing, China; ${ }^{5}$ Wuxi People's Hospital Affiliated to Nanjing Medical University, Wuxi, Jiangsu, China 214023; ${ }^{6}$ Jiangsu Provincial Center for Disease Control and Prevention, Nanjing, Jiangsu, China 210009; ${ }^{7}$ Health Department of Jiangsu Province, Nanjing, Jiangsu, China 210008

\author{
Abstract \\ Objective To determine whether the novel avian influenza H7N9 virus \\ can transmit from person to person and its efficiency. \\ Design Epidemiological investigations conducted after a family cluster \\ of two patients with avian H7N9 in March 2013. \\ Setting Wuxi, Eastern China. \\ Participants Two patients, their close contacts, and relevant \\ environments. Samples from the patients and environments were
}

collected and tested by real time reverse transcriptase-polymerase chain reaction ( $\mathrm{rRT}-\mathrm{PCR}$ ), viral culture, and haemagglutination inhibition assay. Any contacts who became ill had samples tested for avian H7N9 by rRT-PCR. Paired serum samples were obtained from contacts for serological testing by haemagglutination inhibition assays.

Main outcomes measures Clinical data, history of exposure before the onset of illnesses, and results of laboratory testing of pathogens and further analysis of sequences and phylogenetic tree to isolated strains.

Correspondence to: M H Zhou zmh@jscdc.cn and H Wang hua@jscdc.cn

Extra material supplied by the author (see http://www.bmj.com/content/347/bmj.f4752?tab=related\#webextra)

Appendix 1: Supplementary tables and definitions of cases and close contacts

Appendix 2: Phylogenetic trees for eight gene segments

Video on bmj.com (see also http://bmj.com/video)

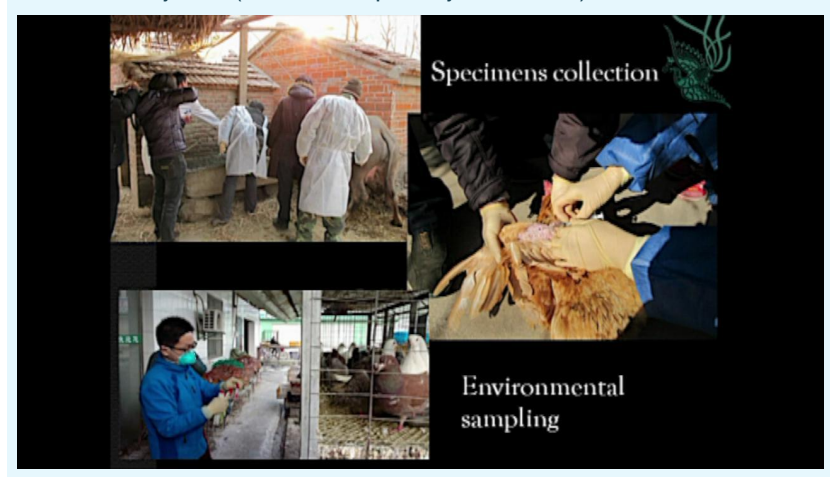


Results The index patient became ill five to six days after his last exposure to poultry. The second patient, his daughter aged 32, who provided unprotected bedside care in the hospital, had no known exposure to poultry. She developed symptoms six days after her last contact with her father. Two strains were isolated successfully from the two patients. Genome sequence and analyses of phylogenetic trees showed that both viruses were almost genetically identical. Forty three close contacts of both patients were identified. One had mild illness but had negative results for avian H7N9 by rRT-PCR. All 43 close contacts tested negative for haemagglutination inhibition antibodies specific for avian H7N9.

Conclusions The infection of the daughter probably resulted from contact with her father (the index patient) during unprotected exposure, suggesting that in this cluster the virus was able to transmit from person to person. The transmissibility was limited and non-sustainable.

\section{Introduction}

A novel avian influenza A (H7N9) virus was identified recently in Eastern China. ${ }^{1}$ As of 30 May 2013, a total of 132 cases were reported, distributed sporadically in 10 provinces/municipalities with one case in Taiwan. ${ }^{2}$ In most of the laboratory confirmed cases the patients developed severe pneumonia and acute respiratory distress syndrome (ARDS) and needed intensive care. ${ }^{3}$ Thirty seven died of respiratory failure or other complications. Human infections with this virus had not been reported before. Infections with other $\mathrm{H} 7$ subtypes, such as $\mathrm{H} 7 \mathrm{~N} 2, \mathrm{H} 7 \mathrm{~N} 3$, and H7N7, which are usually related to outbreaks of poultry, have been reported in several countries. ${ }^{4}$ These subtypes, however, often cause only mild to moderate disease, and only one fatal case has been reported (Netherlands in 2003). ${ }^{5}$

According to the current available epidemiological data, most affected patients had a history of visiting live poultry markets or contact with poultry seven to 10 days before the onset of illnesses, indicating that the sources of infection were likely to be either contaminated environment or infected poultry..$^{6-9}$ Until now, no clear evidence indicated that the novel virus could transmit from person to person. ${ }^{10}$ Family clusters with confirmed or suspected avian $\mathrm{H} 7 \mathrm{~N} 9$ virus infection were previously reported $^{11}$; however, some information related to the cluster in Jiangsu Province was incomplete and even incorrect. Humans seemed to be more susceptible to the H7N9 virus than the H5N1 virus. ${ }^{12}$ It is therefore vital to establish whether the novel virus can transmit from person to person because of the potential threat of a pandemic if it possesses sustainable transmissibility between humans. ${ }^{1314}$ We report a family cluster of two patients with novel avian H7N9 virus infection.

\section{Methods}

\section{Epidemiological investigation and sample collection}

Public health staff interviewed all family members and doctors and nurses who provided service for the two patients on multiple occasions to validate timelines of events and, in particular, to verify possible exposure history before the onset of illnesses, including raising or contact with animals, visiting live poultry markets and purchase of live poultry, and contact with febrile patients. We could not interview the two patients because they were critically ill at the time of investigation.

In addition to the household and surrounding environments (the residential district, which was about 1 million square metres) where the two patients lived, live poultry markets and convenience stores the index patient visited were all inspected to assess potential exposures to poultry and the environment.
We reviewed medical records to determine the time of onset and progression of the illness. All household members and healthcare workers who had contact with the two patients within 1 metre without effective personal protection at any time from the day before illnesses onset to when the patients were isolated in hospitals were treated as close contacts and placed under medical observation for seven days. Paired serum samples (separated by at least three weeks) were collected to ascertain potential person to person transmission as well as asymptomatic and subclinical infections.

Clinical samples for laboratory testing included samples from the two patients on 27 and 31 March, 13 environmental samples including two smears from chicken cages, three smears from pigeon cages, two samples of chicken faeces, one sample of pigeon faeces, one sample of duck faeces, two cloacal swabs from chicken, and two sewer water samples from two live poultry markets on April 2, and two cloacal swabs from swans on 5 April from the residential area where the two patients lived.

\section{Laboratory investigation}

Specimens in viral transport medium were tested by real time reverse transcriptase-polymerase chain reaction (rRT-PCR) analysis and by inoculation into Madin-Darby canine kidney cell (MDCK) culture for viral isolation, including one to three blind passages, as previously described. ${ }^{1}$

Viral RNA was reverse transcribed into cDNA with SuperScript III First-Strand Synthesis System (Invitrogen, Life Technologies, Carlsbad, CA, USA) with random hexamers. Double strand cDNA was synthesised with Sequenase 2.0 (USB, Affymetrix, Cleveland, OH, USA). Sequencing libraries were prepared with the Nextera XT DNA sample preparation Kit (Illumina, San Diego, CA, USA). Samples from the second patient and the environment were pooled together and then run on Illumina MiSeq platform to generate $150 \mathrm{bp}$ paired end reads. Assembly of viral reads was carried out with influenza A isolate A/Anhui/1/2013(H7N9) as the reference genome (GISAID, accessions EPI439503-EPI439510). In addition, full genomes from the index case were amplified with previously described methods ${ }^{15}$ and sequenced on ABI 3130 automatic DNA analyser (Life Technologies) with ABI BigDye Terminator v3.1 cycle sequencing kit (Life Technologies). Full genome sequences of the viruses were deposited in GenBank (accession number: KF034916-KF034923 for the father (index case),

KF034908-KF034915 for the daughter, and KF150605-KF150612 for the environment).

Sequences were compiled with the Lasergene sequence analysis software package (DNAStar, Madison, WI, USA). Nucleotide BLASTn analysis was used to identify related reference strains, and reference sequences were obtained from GeneBank and GISAID. Pairwise sequence alignments were also performed with the MegAlign program (DNASTAR) to determine similarities between nucleotide and amino acid sequence. Phylogenetic analysis of the aligned sequences for eight genomic segments was performed by the maximum likelihood methods with MEGA4.1 software. ${ }^{16}$ The reliability of the unrooted neighbour-joining tree was assessed by bootstrap analysis with 1000 replications; only bootstrap values $\geq 70 \%$ are shown. Horizontal distances are proportional to genetic distance. Alignments of each influenza virus sequence were created with the program ClustalX 1.83 .

Serum samples were tested in a modified in house haemagglutination inhibition assay with turkey erythrocytes. ${ }^{17}$ Antigens for the assays were produced from the 
A/Nanjing/1/2013(H7N9) strain isolated from the confirmed case in Jiangsu. ${ }^{8}$

All testing was performed at the BSL-2 or BSL-3 laboratories of Jiangsu Province Center for Disease Control and Prevention, Nanjing, China.

\section{Results \\ Patients}

The index patient was a retired man aged 60 with a history of hypertension for more than 10 years. He developed a fever, cough, and shortness of breath on 8 March 2013 and was admitted to a Chinese hospital (hospital A) on March 11 with a left upper lobe inflammation. Initial blood routine testing identified no abnormality, except for increased hypersensitive $\mathrm{C}$ reactive protein $(28.5 \mathrm{mg} / \mathrm{L})$. He was treated with azithromycin and piperacillin-sulbactam. Because of progressive respiratory distress, persistent hyperpyrexia, and hypoxemia, he was transferred to the hospital's intensive care unit in the afternoon of 15 March with a diagnosis of viral pneumonitis and type I acute respiratory distress syndrome. He was again transferred to another tertiary hospital's (hospital B) intensive care unit because of deterioration on 18 March and began to treatment with oseltamivir the next day. He did not develop diarrhoea during the course of the disease. He died of disseminated intravascular coagulation and multi-organ failure on 4 May.

The index patient's daughter, an unemployed woman aged 32, was otherwise healthy without any underlying illnesses. She provided bedside care for her father until he was admitted to the second hospital's intensive care unit. She developed fever with body temperature $39.6^{\circ} \mathrm{C}$ and cough on 21 March. On 24 March, she was admitted to the pneumology department of the same hospital (hospital B) with pneumonia in the left upper lobe. Initial testing showed leucocytopenia $\left(2.0 \times 10^{9} / \mathrm{L}\right)$, lymphopenia $\left(0.7 \times 10^{9} / \mathrm{L}\right)$, and slight hypoxia. She was treated with antibiotics (azithromycin and piperacillin-sulbactam). Oseltamivir (75 mg twice a day) was administered on 24 March. She was transferred to intensive care on 28 March because of persistent hyperpyrexia, respiratory failure, and acute respiratory distress syndrome. Although treated with mechanical ventilation, broad spectrum antibiotics, oseltamivir, immunological therapy, and fluid resuscitation, she died of multi-organ failure and cardiac arrest on 24 April. Table A in appendix 1 summarises the clinical characteristics of the two patients.

\section{Epidemiological investigations}

The index patient lived in an elite residential district with his wife, daughter, son in law, and granddaughter. An ornamental pool was located in the centre of the residential district. With the exception of two black swans that were raised by property management personnel for about two years, there were no other fowl or poultry in the residential district. There were two convenience stores on the edge of the residential district located less than 500 metres from the home where only frozen but not live poultry were sold. About $2 \mathrm{~km}$ away from the residential district, there were two free live markets where several kinds of live poultry were sold.

Interviews with the other family members and doctors and nurses who provided services for the two patients permitted reconstruction of a timeline for the index patient and his daughter (figure). $\Downarrow$ The index patient was in charge of purchasing foodstuff for the whole family every day. As a result, he often visited convenience stores and occasionally live markets. In particular, he purchased six quails in one of the live markets and cooked for the family one day between 1 March and 4 March. The exact date was unclear. The second patient was unemployed, and her daily routine was to take care of her daughter aged 22 months. She rarely walked beyond her residential district. From the time the index patient became ill until he was admitted to the second hospital's intensive care unit, a period of about 10 days, the daughter provided much of the care for her father between 8 and 10 March at home (conventional care) and in the afternoons between 11 and 13 March (visiting), all day on 14 March (bedside care), and in the morning of 15 March (bedside care) in the hospital, including bedside care for six to eight hours a day. From 14 March, her father had abundant expectoration. More importantly, aside from conventional care, she was in charge of cleaning her father's secretions and maintaining his oral hygiene during care without any personal protective equipment. The son in law provided bedside care for the index patient during other time periods. The father was transferred to intensive care in the afternoon of 15 March. After then, she visited her father once a day for about half an hour in the afternoons from 16 to 18 March wearing a mask and had no close contact with her father after 18 March because he was transferred to an isolated single unit, where she visited her father only through a glass window.

We drew up a full list of potential contacts, including everyone who had contact with the patients from the onset of their illnesses till effective isolation on 2 April, and local public health officials then interviewed individuals. We identified 43 close contacts, including 39 healthcare workers, three household members, and one relative. Among the 39 healthcare workers, 15 came from hospital A and were close contacts of the index case; 24 came from hospital B; 14 were close contacts of the daughter, and 10 were close contacts of both patients (see table $\mathrm{B}$ in appendix 1). The main types of contact for patients were physical examination and various medical services. Contact with patients took place at least once a day. The mean number of days of contact with patients was four (range 1-8 days). Other than the husband of the second case, who had a slight fever $\left(37.5^{\circ} \mathrm{C}\right)$ on $31 \mathrm{March}$, no other close contacts developed febrile respiratory symptoms. He was treated with oseltamivir, and his throat swab sample tested negative for influenza A viruses by rRT-PCR with universal primers. All other 42 close contacts did not receive prophylactic antiviral drug after exposure. No haemagglutination inhibition antibodies against $\mathrm{A}$ (H7N9) virus were detected from paired serum samples of all close contacts. Higher titres of haemagglutination inhibition antibodies, however, were observed from both patients (figure). $\Downarrow$

\section{Laboratory investigations}

rRT-PCR analysis of an endotracheal aspirate specimen collected on 31 March from the index patient indicated that it contained weak novel avian H7N9 nucleic acid, although no positive results were obtained from a throat swab sample collected on 27 March. Both throat swab and endotracheal aspirate samples collected on 27 March and 31 March from the daughter were also positive for avian H7N9 viral genes. Infection with other respiratory pathogens, such as SARS-CoV, novel $\mathrm{CoV}$, seasonal influenza viruses (classic $\mathrm{H} 1$, novel H1pdm09, H3, or B), and avian influenza H5N1 and H9N2, was not detected.

Three strains-two from samples collected on 31 March from the index and subsequent patient (figure $\Downarrow$ ) and one from a smear sample forma poultry cage collected on 2 April from one of the two live poultry markets that the index patient often visited-were isolated successfully. Sequences of all eight genes 
of the two strains isolated from patients were nearly identical (similarity ranged from $99.6 \%$ to $99.9 \%$ ) but slightly different from the strain isolated from environment (table) $\Downarrow$. In addition, sequencing of the two strains from patients showed that all the viral genes were avian and were closely related to other human A (H7N9) viral sequences previously identified in Shanghai, Zhejiang, and Anhui Province but genetically different from strains isolated from the environment and birds. Further phylogenetic analysis showed all eight genes of the two strains from patients belonged to the same clade (see appendix 2).

Analysis of the key functional amino acid sites in different viral proteins associated with interspecies transmission or drug resistance of the three isolates showed that both strains isolated from patients were the same as the strains of Shanghai/2, Anhui/1, and Nanjing/1 but somewhat different from strain of Shanghai/1, which had R294K of NA and isoleucine (I) but not valine (V) at 368 site of PB1. In addition, the two strains isolated from the patients had $\mathrm{E} 627 \mathrm{~K}$; however, the strain isolated from environment still possessed $\mathrm{E}$ at the same site (see table $\mathrm{C}$ in appendix 1).

\section{Discussion}

We believe that the most likely explanation for this family cluster of the two patients with novel avian influenza H7N9 virus infection is that the virus transmitted directly from the index patient to his daughter. Firstly, the diagnosis of the two patients was confirmed virologically, and the clinical features $\square$ fever, pneumonia with lymphopenia, and rapid progression to acute respiratory distress syndrome-all correspond to the cardinal features of reported cases in humans in China. ${ }^{17}{ }^{18}$ Secondly, it was fortuitous for the investigation that the daughter did not visit the markets to buy foodstuffs and cook for the family and had no exposure to animals or history of visiting live poultry markets. She did, however, have prolonged, direct, and unprotected exposure to her father. Thirdly, two strains were isolated successfully from the two patients. Further sequence analysis showed that both possessed high degrees of similarity between nucleotide (99.6\%-99.9\%) and amino acid (99.0\%-100\%) sequences. Finally, before this study, A (H7N7) among H7 subtype influenza viruses and H5N1 virus was known to have the ability to transmit from person to person. ${ }^{19-21}$ Animal (ferrets and pigs) experiments indicated that the H7N9 virus possessed the capability to bind to both avian and human receptor and to transmit itself by droplet under certain conditions. ${ }^{22} 23$

Our findings, however, indicated that the virus has not gained the ability for efficient sustained transmission from person to person. From 14 March, the daughter came into direct contact with the oral secretions of the index patient without any personal protective equipment, contacting the patient at a much higher rate than other individuals in contact with the patient. Similar to other available human strains, the characteristics of the two strains showed no adaptation change in the receptor binding site from the avian 2,3-linked pattern toward the human 2,6-linked pattern of sialic acid receptor. ${ }^{17}$ Phylogenic tree analysis of all eight genomic segments indicated that the two isolates were of avian origin and that there was no reassortment with human or swine influenza viruses. ${ }^{24-26}$ Furthermore, no asymptomatic or subclinical infections were identified among 43 close contacts by haemagglutination inhibition testing. Recent studies also indicated that avian H7N9 tends to bind lower pulmonary epithelial cells rather than those of the upper respiratory tract, which makes it difficult to transmit between humans. $^{7}$
The two patients were blood related. The index patient's son in law also provided bedside care for the index patient in the mornings and nights between 11 and 13 March without any personal protective equipment. Both biological and serological evidence showed that he was not infected with the H7N9 virus. These findings suggest that potential genetic susceptibility might be one of determinants and that avian influenza viruses, like $\mathrm{H} 5 \mathrm{~N} 1$, are more easily transmitted between individuals with genetic connection. ${ }^{27} 28$

The possible source of infection for the index case was likely to be from the live poultry market that the patient used to visit or the six quails that he bought but were slaughtered by the seller in the market one week before illness onset. One strain of avian $\mathrm{H} 7 \mathrm{~N} 9$ was isolated from environmental samples from the live poultry markets. Visiting a wet poultry market has been identified as a risk factor for human infections. ${ }^{89}$

\section{Important strengths and differences in relation to other studies}

With respect to the possible infection period in the second patient, we cannot ascertain when person to person transmission occurred. The most likely period was from 11 to 15 March during the index patient's admission to hospital before transfer to intensive care, especially the periods between the afternoon of 14 March and the morning of 15 March, when the index patient began to expectorate abundantly. The daughter had direct and close contact with the index case without any protection during this period. During 8-10 March, the daughter provided only conventional care for the index patient, such as taking his temperature. Transmission is unlikely to have occurred during this period as the patient had not yet to start expectoration. Thus, the most likely incubation period was six to seven days (range 6-13 days) based on the daughter's unprotected exposure to her father. The putative incubation period was a relatively longer than that reported by Cowling and colleagues, who estimated the average incubation period to be around three days based on Weibull model as well as live poultry to human transmission. ${ }^{29}$ We estimated the incubation period based on person to person transmission and one case in cluster.

\section{Implications of the study}

Possible transmission routes include contact while cleaning up infected oral secretions and subsequent inoculation of mucous membranes or the respiratory tract. Some researchers suggested that the daughter might have acquired her infection during the process of washing her father's diarrhoea-soiled underwear. ${ }^{11}$ We thought this to be unlikely. Firstly, clinical data showed that the index patient did not develop diarrhoea during course of disease. Secondly, the daughter wore gloves while washing. Thirdly, no nucleic acids specific for avian H7N9 were detected from the faecal samples from the father.

We noted that 39 healthcare workers were identified as close contacts in this cluster, which was a little unusual. Both the two hospitals where the two patients were admitted were general hospitals rather than hospitals specialising in infectious disease. The awareness of personal protection of the healthcare workers was relatively weak. They used common surgical masks instead of N95 masks while providing medical services for the two patients. Another important factor was the relatively long time between the patients' admission to a clear diagnosis with H7N9 virus infection. After the cluster was identified, infection control measures were initiated to prevent potential nosocomial transmission. Patients were isolated. Healthcare workers were required take standard respiratory and contact protection. 


\section{Weaknesses of the study}

There are several limitations to our study. Firstly, we could not interview with the two patients as they were both critically ill. Therefore, the possibility that the daughter acquired her infection from the environment or other sources could not be completely ruled out, although we believe that it is less likely.

Environmental investigation discovered that in addition to two swans raised by an employee of the property management, there were no other birds or poultry in her living environment. No positive results were detected from the cloacal swab and faecal samples from the two swans. Secondly, the sensitivity of haemagglutination inhibition assay was not satisfied. Thus, subclinical or asymptomatic infections could not be excluded among close contacts. A more sensitive method such as micro-neutralisation assay should be considered in the future. Finally, whole sequence alignment showed that the two isolates from patients were not identical. It is reasonable to expect slight differences between the two strains. Variations of the viral genomes occur constantly because of the high error rate of the viral polymerase. We sequenced viral genomes from the strain from the father that underwent three successive passages using MDCK cell lines. Evolution of the strain might have occurred in vivo because of the long time interval as well as the use of antiviral drugs. A previous study showed that influenza viruses often differ from those present in clinical specimens after isolation in MDCK cell culture as adaptive changes occur during virus transmission from the human host to cells of heterologous origin. ${ }^{30}$

\section{Unanswered questions and future research}

To the best of our knowledge, this is the first report of probable transmissibility of this novel virus from person to person with detailed epidemiological, clinical, and virological data. The importance of an isolated case of such transmission means there is potential for greater human to human transmission. Thus, timely detection as well as rapid investigation and risk assessments of clusters is critically important as the increase in clusters might indicate potential transmissibility of a novel virus.

We thank the of staff at Jiangsu Provincial and Wuxi Municipal CDC, Binhu District CDC, for their help in field investigation and collection of environmental samples; Mike Zhongyu He from Krieger School of Arts and Sciences, Johns Hopkins University; and Johanna Lam from Frances Paynebolton School of Nursing, Case Western Reserve University.

Contributors: $\mathrm{HW}$ and $\mathrm{M}-\mathrm{HZ}$ were co-principals who designed and supervised the study. C-JB, Y-HQ, HJ, YH, F-YT, BL, KX, and CS were in charge of field investigation, clinical data, and sample collection. $X Q$, $X-L G$, and L-BC performed the laboratory testing including rRT-PCR and haemagglutination inhibition assays, genome sequence, and phylogenetic tree analysis. C-JB, XQ, and L-BC drafted the manuscript and other co-authors contributed to review and comment and approved the final version. XQ, Y-HQ, C-JB, X-LG, and L-BC contributed equally to this study. C-J B and HW are guarantors.

Funding: $\mathrm{HW}$ and $\mathrm{M}-\mathrm{HZ}$ are partly supported by the Innovation Platform for Public Health Emergency Preparedness and Response (No ZX201109). XQ and L-BC are partly supported by the Jiangsu Province Key Medical Talent Foundation (RC2011084 and RC2011191) and the " 333 " Projects of Jiangsu Province. C-JB is partly supported by Jiangsu Province Science and Technology Support Program (social development: BE2012769). Y-HQ is partly supported by Jiangsu Provincial Project of Preventive Medicine (Y201026) and Wuxi Development Project of Science and Technology Bureau (CSZ00955, CSZ01051). The funding bodies had no role in study design, data collection and analysis, preparation of the manuscript, or the decision to publish. The contents of this article are solely the responsibility of the authors and do not necessarily represent the views of the Jiangsu Provincial Centers for Disease Control and Prevention or other organisations.

Competing interests: All authors have completed the ICMJE uniform disclosure form at www.icmje.org/coi_disclosure.pdf (available on request from the corresponding author) and declare: no support from any organisation for the submitted work; no financial relationships with any organisations that might have an interest in the submitted work in the previous three years; no other relationships or activities that could appear to have influenced the submitted work.

Ethical approval: An ethics waiver was granted and authorised under National Emergent Public Health Events Act. According to this Act, collection of data related to H7N9 cases was an important part in epidemic analyses and subsequent control measures. Therefore, the investigation was exempt from institutional board assessment.

Data sharing: No additional data available.

1 Gao R, Cao B, Hu Y, Feng Z, Wang D, Hu W, et al. Human infection with a novel avian-origin influenza A (H7N9) virus. N Engl J Med 2013;368:1888-97.

2 Human infection with influenza A(H7N9) virus in China-update. World Health Organization, 2013

3 Gao HN, Lu HZ, Cao B, Du B, Shang H, Gan JH, et al. Clinical findings in 111 cases of influenza A (H7N9) virus infection. N Engl J Med 2013;368:2277-85.

4 Belser JA, Bridges CB, Katz JM, Tumpey TM. Past, present, and possible future human infection with influenza virus A subtype H7. Emerg Infect Dis 2009;15:859-65.

5 Fouchier RA, Schneeberger PM, Rozendaal FW, Broekman JM, Kemink SA, Munster V, et al. Avian influenza A virus (H7N7) associated with human conjunctivitis and a fatal case of acute respiratory distress syndrome. Proc Natl Acad Sci U S A 2004;101:1356-61.

6 Shi J, Deng G, Liu P, Zhou J, Guan L, Li W, et al. Isolation and characterization of H7N9 viruses from live poultry markets-implication of the source of current H7N9 infection in humans. Chinese Science Bulletin 2013;58:1857-63.

7 Chen Y, Liang W, Yang S, Wu N, Gao H, Sheng J, et al. Human infections with the emerging avian influenza A H7N9 virus from wet market poultry: clinical analysis and characterisation of viral genome. Lancet 2013;381:1916-25.

8 Bao CJ, Cui LB, Zhou MH, Hong L, Gao GF, Wang H. Live-animal markets and influenza A (H7N9) virus infection. N Engl J Med 2013;368:2337-9.

9 Li J, Yu X, Pu X, Xie L, Sun Y, Xiao H, et al. Environmental connections of novel avian-origin H7N9 influenza virus infection and virus adaptation to the human. Sci China Life Sci 2013;56:485-92.

10 Uyeki TM, Cox NJ. Global concerns regarding novel influenza A (H7N9) virus infections. N Engl J Med 2013;368:1862-4.

11 Li Q, Zhou L, Zhou M, Chen Z, Li F, Wu H, et al. Preliminary report: epidemiology of the avian influenza a (h7n9) outbreak in China. N Engl J Med 2013 April 24, epub ahead of print.

12 Parry J. H7N9 virus is more transmissible and harder to detect than H5N1, say experts. BMJ 2013;346:f2568.

13 From SARS to H7N9: will history repeat itself? Lancet 2013;381:1333.

14 Horby P. H7N9 is a virus worth worrying about. Nature 2013:496:399.

15 Hoffmann E, Stech J, Guan Y, Webster RG, Perez DR. Universal primer set for the full-length amplification of all influenza A viruses. Arch Virol 2001;146:2275-89.

16 Tamura K, Dudley J, Nei M, Kumar S. MEGA4: molecular evolutionary genetics analysis (MEGA) software version 4.0. Mol Biol Evol 2007;24:1596-9.

17 Laboratory Procedures-serological detection of avian influenza A(H7N9) virus infections by turkey haemagglutination-inhibition assay. World Health Organization, 2013.

$18 \mathrm{Lu} \mathrm{SH}, \mathrm{Xi}$ XH, Zheng YF, Cao Y, Liu XN, Lu HZ. Analysis of the clinical characteristics and treatment of two patients with avian influenza virus (H7N9). Biosci Trends 2013;7:109-12.

19 Du Ry van Beest Holle M, Meijer A, Koopmans M, de Jager CM. Human-to-human transmission of avian influenza A/H7N7, The Netherlands, 2003. Euro Surveill 2005;10:264-8.

20 Wang H, Feng Z, Shu Y, Yu H, Zhou L, Zu R, et al. Probable limited person-to-person transmission of highly pathogenic avian influenza A (H5N1) virus in China. Lancet 2008;371:1427-34

21 Ungchusak K, Auewarakul P, Dowell SF, Kitphati R, Auwanit W, Puthavathana P, et al. Probable person-to-person transmission of avian influenza A (H5N1). N Engl J Med 2005;352:333-40.

22 Zhu H, Wang D, Kelvin DJ, Li L, Zheng Z, Yoon SW, et al. Infectivity, transmission, and pathology of human-isolated H7N9 influenza virus in ferrets and pigs. Science 2013;341:183-6.

23 Zhang Q, Shi J, Deng G, Guo J, Zeng X, He X, et al. H7N9 influenza viruses are transmissible in ferrets by respiratory droplet. Science 2013 July 18 , epub ahead of print.

24 Kageyama T, Fujisaki S, Takashita E, Xu H, Yamada S, Uchida Y, et al. Genetic analysis of novel anvian $A(H 7 N 9)$ influenza viruses isolated from patients in China, February to April 2013. Euro Surveill 2013;18:20453.

25 Liu D, Shi W, Shi Y, Wang D, Xiao H, Li W, et al. Origin and diversity of novel avian influenza A H7N9 viruses causing human infection: phylogenetic, structural, and coalescen analyses. Lancet 2013;381:1926-32.

26 Liu Q, Lu L, Sun Z, Chen GW, Wen Y, Jiang S. Genomic signature and protein sequence analysis of a novel influenza $A$ (H7N9) virus that causes an outbreak in humans in China. Microbes Infect 2013;15:432-9.

27 Abdel-Ghafar AN, Chotpitayasunondh T, Gao Z, Hayden FG, Nguyen DH, de Jong MD, et al. Update on avian influenza A (H5N1) virus infection in humans. N Engl J Med 2008;358:261-73.

28 Horby P, Nguyen NY, Dunstan SJ, Baillie JK. The role of host genetics in susceptibility to influenza: a systematic review. PLoS One 2012;7:e33180. 


\section{What is already known on this topic}

Most cases of novel avian H7N9 occur sporadically

Animal experiments indicated that the H7N9 virus can transmit itself by droplet under certain conditions

No definite evidence indicates that the novel virus can transmit itself from person to person

\section{What this study adds}

To our best knowledge, this is the first report of probable transmissibility of the H7N9 virus from person to person with detailed epidemiological, clinical, and virological data

Our findings reinforced that the novel virus possesses the potential for pandemic spread

29 Cowling BJ, Jin L, Lau EH, Liao Q, Wu P, Jiang H, et al. Comparative epidemiology of human infections with avian influenza A H7N9 and H5N1 viruses in China: a population-based study of laboratory-confirmed cases. Lancet 2013;382:129-37.

30 Zhirnov OP, Vorobjeva IV, Saphonova OA, Poyarkov SV, Ovcharenko AV, Anhlan D, et al. Structural and evolutionary characteristics of HA, NA, NS and M genes of clinical influenza A/H3N2 viruses passaged in human and canine cells. J Clin Virol 2009;45:322-33.

Accepted: 24 July 2013

\section{Cite this as: BMJ 2013;347:f4752}

This is an Open Access article distributed in accordance with the Creative Commons Attribution Non Commercial (CC BY-NC 3.0) license, which permits others to distribute, remix, adapt, build upon this work non-commercially, and license their derivative works on different terms, provided the original work is properly cited and the use is non-commercial. See: http://creativecommons.org/licenses/by-nc/3.0/. 


\section{Table}

Table 1/ Nucleotide sequences identities among eight genes of three strains ${ }^{*}$ isolated from two patients and environment related to family cluster of novel avian influenza A (H7N9) virus infection in Eastern China, 2013

\begin{tabular}{|c|c|c|c|}
\hline \multirow[b]{2}{*}{ Gene segment divergence } & \multicolumn{3}{|c|}{ Percent identity } \\
\hline & Wuxi/1 & Wuxi/2 & Env/1 \\
\hline \multicolumn{4}{|l|}{ PB2 } \\
\hline Wuxi/1 & - & 99.9 & 99.7 \\
\hline Wuxi/2 & 0.1 & - & 99.6 \\
\hline Env/1 & 0.3 & 0.4 & - \\
\hline \multicolumn{4}{|l|}{ PB1 } \\
\hline Wuxi/1 & - & 99.9 & 99.4 \\
\hline Wuxi/2 & 0.1 & - & 99.3 \\
\hline Env/1 & 0.6 & 0.7 & - \\
\hline \multicolumn{4}{|l|}{ PA } \\
\hline Wuxi/1 & - & 99.8 & 99.8 \\
\hline Wuxi/2 & 0.2 & - & 99.8 \\
\hline Env/1 & 0.2 & 0.2 & - \\
\hline \multicolumn{4}{|l|}{ NP } \\
\hline Wuxi/1 & - & 99.9 & 99.7 \\
\hline Wuxi/2 & 0.1 & - & 99.7 \\
\hline Env/1 & 0.3 & 0.3 & - \\
\hline \multicolumn{4}{|l|}{ HA } \\
\hline Wuxi/1 & - & 99.9 & 99.7 \\
\hline Wuxi/2 & 0.1 & - & 99.6 \\
\hline Env/1 & 0.3 & 0.4 & - \\
\hline \multicolumn{4}{|l|}{ NA } \\
\hline Wuxi/1 & - & 99.6 & 99.1 \\
\hline Wuxi/2 & 0.4 & - & 99.4 \\
\hline Env/1 & 0.9 & 0.6 & - \\
\hline \multicolumn{4}{|l|}{$\mathbf{M}$} \\
\hline Wuxi/1 & - & 99.9 & 97.9 \\
\hline Wuxi/2 & 0.1 & - & 97.8 \\
\hline Env/1 & 2.1 & 2.2 & - \\
\hline \multicolumn{4}{|l|}{ NS } \\
\hline Wuxi/1 & - & 99.6 & 99.5 \\
\hline Wuxi/2 & 0.4 & - & 99.4 \\
\hline Env/1 & 0.5 & 0.6 & - \\
\hline
\end{tabular}

${ }^{*} \mathrm{~A} /$ Wuxi/1/2013 (Wuxi/1) isolated from daughter, A/Wuxi/2/2013 (Wuxi/2) isolated from index patient (father), and A/Environment/Wuxi/1/2013 (Env/1) isolated from environmental sample collected from free live market where index patient used to visit. 


\section{Figure}

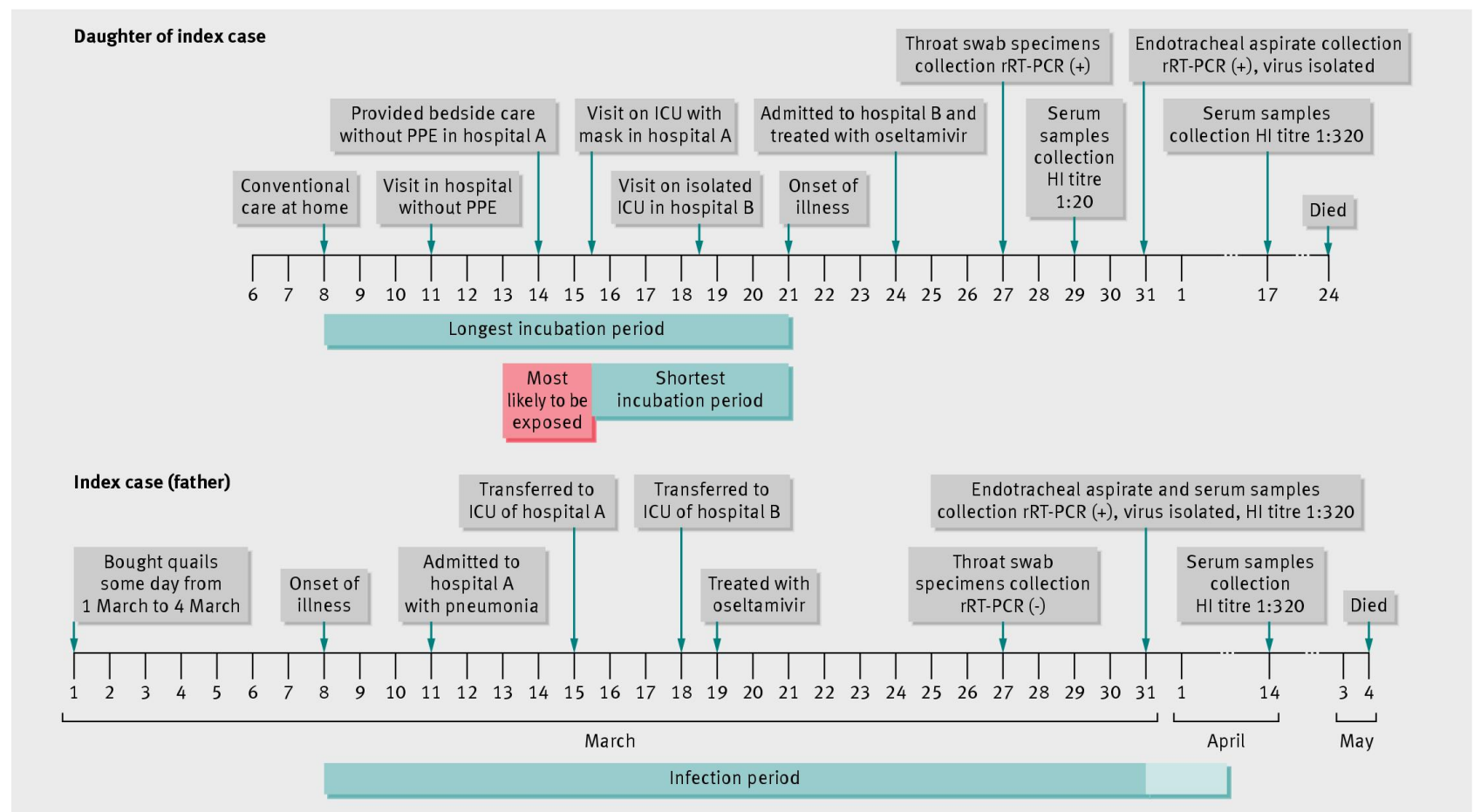

Timeline of family cluster of two patients with novel avian influenza A (H7N9) virus infection in Eastern China, 2013. Strain isolation used Madin-Darby canine kidney cell culture (rRT-PCR=real time reverse transcriptase-polymerase chain reaction; $\mathrm{HI}=$ haemagglutination inhibition) 